\title{
Effect of pretreatment methods on the synergism of cellulase and xylanase during the hydrolysis of bagasse
}

Lili Jia ${ }^{\mathrm{a}}$, Geisa A. L. Gonçalves ${ }^{\mathrm{a}}$, Yusaku Takasugi ${ }^{\mathrm{a}}$, Yutaro Mori ${ }^{\mathrm{a}}$, Shuhei Noda ${ }^{\mathrm{b}}$, Tsutomu Tanaka $^{\mathrm{c}}$, Hirofumi Ichinose ${ }^{\mathrm{d}}$, Noriho Kamiya ${ }^{\mathrm{a}, \mathrm{e}, *}$

${ }^{a}$ Department of Applied Chemistry, Graduate School of Engineering, Kyushu University, 744 Motooka, Fukuoka 819-0395, Japan

${ }^{b}$ Biomass Engineering Program, RIKEN, 1-7-22, Suehiro-cho, Tsurumi-ku, Yokohama, Kanagawa 230-0045, Japan

${ }^{\mathrm{c}}$ Department of Chemical Science and Engineering, Graduate School of Engineering, Kobe University, 1-1 Rokkodaicho, Nada, Kobe 657-8501, Japan

${ }^{\mathrm{d}}$ Faculty of Agriculture, Kyushu University, Hakozaki 6-10-1, Higashi-ku, Fukuoka 812-8581, Japan

${ }^{\mathrm{e}}$ Center for Future Chemistry, Kyushu University, Fukuoka 819-0388, Japan

*Corresponding author: Tel.: (+81) 092-802-2807; fax: (+81) 092-802-2810, nori_kamiya@mail.cstm.kyushu-u.ac.jp

Other authors: L. Jia lilijia@mail.cstm.kyushu-u.ac.jp G.A.L. Gonçalves geisa@mail.cstm.kyushu-u.ac.jp, Y. Takasugi y_takasugi@mail.cstm.kyushu-u.ac.jp, Y. Mori forest@mail.cstm.kyushu-u.ac.jp, S. Noda shuhei.noda@riken.jp, T. Tanaka tanaka@kitty.kobe-u.ac.jp, H. Ichinose ichinose@agr.kyushu-u.ac.jp 


\begin{abstract}
The effect of pretreatment with peracetic acid (PAA) or an ionic liquid (1-ethyl-3-methylimidazolium acetate, $[$ Emim $][\mathrm{OAc}])$ on the synergism between endoglucanase and endoxylanase in the hydrolysis of bagasse was investigated. An endoglucanase, Cel6A, with a carbohydrate-binding module (CBM) and two endoxylanases, XynZ-C without a CBM and Xyn11A with an intrinsic xylan/cellulose binding module (XBM), were selected. The hemicellulose content, especially arabinan, and the cellulose crystallinity of bagasse were found to affect the cellulase-xylanase synergism. More specifically, higher synergism (above 3.4) was observed for glucan conversion, at low levels of arabinan (0.9\%), during the hydrolysis of PAA pretreated bagasse. In contrast, [Emim][OAc] pretreated bagasse, showed lower cellulose crystallinity and achieved higher synergism (over 1.9) for xylan conversion. Ultimately, the combination of Cel6A and Xyn11A resulted in higher synergism for glucan conversion than the combination of Cel6A with XynZ-C, indicating the importance of the molecular architecture of enzymes for metabolic synergism.
\end{abstract}

Keywords: Pretreatment; Hemicellulose; Synergism; Endoglucanase; Endoxylanase 


\section{Introduction}

Second-generation bioethanol, produced from agriculture residues, as a substitute for fossil fuels has received widespread attention since the end of the last century. Owing to the prior abundance and appreciable polysaccharides percentage, sugarcane bagasse is considered an ideal substrate for biorefineries (Chandel et al., 2012). However, because of the complex matrix of polysaccharides and lignin within the structure of the plant cell wall, pretreatment becomes an indispensable step to accelerate the degradation of the lignin-carbohydrate complex and to ensure that bioethanol production is a competitive and sustainable process (Davis et al., 2013).

Studies aimed at improving monosaccharide production from sugarcane bagasse include the use of acid, base, hydrogen peroxide and steam explosive processes (Lv et al., 2013; Sant'Ana da Silva et al., 2011). Previous studies showed that peracetic acid (PAA) has good delignification ability, because PAA generates the hydroxonium ion, $\mathrm{HO}^{+}$, which attacks the electron sites of lignin related structure via an electrophilic reaction (Kumar et al., 2013; Uju et al., 2013; Zhao et al., 2007). Ionic liquids (IL) as emerging reagents for biomass pretreatment have been extensively studied because of their excellent characteristics and unique cellulose dissolution capability (Brandt et al., 2013; Mora-Pale et al., 2011). This dissolution is induced by the formation of the electron donor-acceptor complex between the anion of IL and the free hydroxyl group on cellulose, and between the cation of IL and the hydroxyl oxygen atoms on the cellulose chain. Among the different ILs, [Emim][OAc] has proven to be one of the most effective in solubilizing cellulose (Brandt et al., 2013).

Naturally, xylans are highly cross-linked with cellulose fibrils by diferulic bridges, contributing to the firm cell wall matrix (Saha, 2003). As a result, access to cellulose by 
cellulase is generally prevented and this reduction in cellulose activity can hamper downstream successive enzymatic hydrolysis processes. Nonetheless, hemicellulose is a desired polysaccharide that can also be yielded from biomass conversion. The addition of accessory enzymes, especially xylanases, to degrade the hemicellulose content is an alternative approach to make cellulose more accessible to cellulases, and to yield more saccharides simultaneously. Thus, the synergistic use of xylanases with cellulases has become an attractive approach for cellulose degradation. Research using commercial enzymes, which contain a consortium of enzymes, have demonstrated the synergism between cellulases and xylanases (Kumar and Wyman, 2009a; Li et al., 2014; Zhang and Viikari, 2014). In contrast, there are specific efforts that have focused on the synergism between pure enzyme components. Selig and co-workers have demonstrated a significant increase in the depolymerization of hot water treated corn stover by cellobiohydrolase I (CBHI) with the addition of endoxylanase and/or other accessory enzymes (ferulic acid esterase, acetyl xylan esterase) (Selig et al., 2008). A recent report has shown the synergy between CBHI (Cel7A), endoxylanases (GH10 and GH11) and/or xyloglucanase (GH5) during hydrolysis of steam pretreated lignocellulosic biomass substrates; however, the reaction efficiency was highly substrate specific (Hu et al., 2013).

Previous work has shown the use of bagasse as a substrate to study the synergistic effect between cellulase and xylanase. High synergy (6.3) between endoxylanase Xyn11A from Cellulomonas flavigena and endoglucanase Cel7B from Trichoderma reesei for reducing sugars production has been reported during the hydrolysis of alkaline pretreated bagasse (Pavón-Orozco et al., 2012). Nonetheless, a recent report using four types of reagents to pretreat bagasse observed distinct synergism between 
cellulase and xylanase. Steam exploded bagasse, $\mathrm{NaOH}$ and $\mathrm{H}_{2} \mathrm{O}_{2}$ pretreated bagasse showed obvious synergy between cellulase and xylanase for glucose and xylose production, whereas $\mathrm{H}_{2} \mathrm{SO}_{4}$ pretreated bagasse did not (Li et al., 2014). Thus, it appeared that synergism between cellulase and xylanase was specific to the substrate and the pretreatment method used.

In the present work, the physical structure and chemical composition of bagasse after PAA and [Emim][OAc] pretreatments were determined to verify the potential synergistic effect between endoglucanase and endoxylanase. For rationalizing the action of the hydrolases with different catalytic properties, one endoglucanase, Cel6A (GH6, with a CBM) from Thermobifida fusca, and two different endoxylanases, XynZ-C (GH10, without a CBM) from Clostridium thermocellum and Xyn11A (GH11, with a XBM) from T. fusca, were selected. The performance of each enzyme with varied molecular structure in the hydrolysis of pretreated bagasse with different chemical and physical properties was systematically investigated.

\section{Materials and methods}

\subsection{Materials and enzymes}

The biomass bagasse with an average particle size of $200 \mu \mathrm{m}$ was purchased from Toyota Tsusho Corporation (Nagoya, Japan). 1-Ethyl-3-methylimidazolium acetate ([Emim][OAc]) was purchased from Kanto Kagaku, Tokyo, Japan. PAA was supplied by the Mitsubishi Gas Chemical Company, Inc., Japan. The details of the expression and purification of enzymes used in this study are provided in the supplementary information. The enzyme purities were confirmed by sodium dodecyl sulfate polyacrylamide gel electrophoresis (Fig. S1). 


\subsection{Biomass pretreatments and regeneration}

Fifty milligrams of bagasse was added to $1 \mathrm{~g}$ [Emim][OAc] or $1 \mathrm{~mL}$ deionized water that includes $50 \mu \mathrm{L}$ of a $40 \%(w / w)$ PAA solution. The mixture was heated at $80{ }^{\circ} \mathrm{C}$ with stirring (200 rpm) for $3 \mathrm{~h}$. After the reaction, $5 \mathrm{~mL}$ deionized water was added into the tubes to precipitate the regenerated cellulose with vigorous shaking. Subsequently, the regenerated bagasse and solution were separated by centrifugation at $25^{\circ} \mathrm{C}(5,800 \mathrm{~g}, 20 \mathrm{~min})$. The regenerated water was kept at $-18{ }^{\circ} \mathrm{C}$ for further investigation. In order to remove the residual reagent, the regenerated cellulose was washed with $5 \mathrm{~mL}$ deionized water three times. The regenerated cellulose was then dried by lyophilization overnight. All pretreatment experiments were carried out in triplicates. The dry mass of the regenerated cellulose was weighed and the yield of the regenerated biomass was calculated by the following equation (Uju et al., 2013):

$$
Y R B(\%)=M_{\text {pretreated biomass }} / M_{\text {initial biomass }} \times 100 \%
$$

\subsection{Chemical composition of bagasse and sugar loss in regenerated water during the}

\section{pretreatment process}

The chemical composition of untreated, PAA pretreated and [Emim][OAc] pretreated bagasse was measured following the protocol (LAP TP-510-42619, 42618, 42622) from the National Renewable Energy Laboratory (Sluiter et al., 2011, 2008a, 2008b). Measuring sugar loss in regenerated water was performed with small modifications. Briefly, the samples were treated with $72 \%$ sulfuric acid at $30{ }^{\circ} \mathrm{C}$ for $1 \mathrm{~h}$, then diluted to $4 \%$ and incubated in an autoclave at $121^{\circ} \mathrm{C}$ for $1 \mathrm{~h}$. After cooling the 
sample, calcium carbonate was used for neutralizing the $\mathrm{pH}$ to 7 . Analysis of the sugar content was performed using a high-performance liquid chromatography (HPLC) system equipped with a Shodex sugar KS-801 column $(8.0 \times 300$ mm, Showa Denko Co., Tokyo, Japan) and an RI detector at $80{ }^{\circ} \mathrm{C}$ with HPLC-grade water as the eluent at a flow rate of $1 \mathrm{~mL} / \mathrm{min}$. The remaining acid insoluble lignin was measured after oven-drying overnight at $45{ }^{\circ} \mathrm{C}$ and the amount of acid soluble lignin was determined by measuring the absorbance at $240 \mathrm{~nm}$ against a deionized water blank using Jasco UV-Vis spectrophotometer V-550 (Jasco, Tokyo, Japan).

\subsection{Analytical methods}

Scanning electron microscopy (SEM) was employed to observe the changes in the surface of bagasse before and after PAA and [Emim][OAc] pretreatments. The equipment, Shimadzu SS-550 scanning electron microscope (Shimadzu Co., Kyoto, Japan), was operated at $40 \mathrm{kV}$ to image the samples. X-ray diffraction (XRD) measurements were conducted using a Rigaku MultiFlex Diffractometer (Rigaku Co., Tokyo, Japan) operating at $40 \mathrm{kV}$ and $30 \mathrm{~mA}$. The samples were scanned in a $2 \theta$ range from 5 to $50^{\circ}$ using steps of $0.02^{\circ}$. The calculation of cellulose crystallinity $(\mathrm{CCr})$ was derived from the XRD spectra according to Xu et al. (2013):

$$
C C r=\left(I_{002}-I_{\mathrm{am}}\right) / I_{002} / C \times 100 \%
$$

where $C C r$ is the cellulose crystallinity, $I_{002}$ is the maximum intensity of the (002) lattice diffraction, $I_{\mathrm{am}}$ is the peak of the amorphous part evaluated as the minimum intensity between (101) and (002) lattice planes and $C$ is the cellulose percentage of the biomass. 


\subsection{Enzymatic hydrolysis and sugars analysis of hydrolysates}

The hydrolysis experiments were carried out with $0.25 \mathrm{wt} \%$ of the biomass at $50{ }^{\circ} \mathrm{C}$ and 1,000 rpm, in $2 \mathrm{~mL}$ of sodium phosphate buffer (50 mM, pH 7.0). In each tube the total enzyme loading was held constant at $2 \mathrm{mg}$ protein/g biomass. For the binary mixtures, each enzyme loading was $1 \mathrm{mg} / \mathrm{g}$, and for the ternary mixture, $1 \mathrm{mg} / \mathrm{g}$ Cel6A was supplemented with $0.5 \mathrm{mg} / \mathrm{g}$ XynZ-C and $0.5 \mathrm{mg} / \mathrm{g}$ Xyn11A. One hundred microliter samples were taken at $0,3,6,12,24,48$ and $72 \mathrm{~h}$ time points and were analyzed for reducing sugars (glucose as the standard) by the dinitrosalicylic acid method (Miller, 1959). The samples after $72 \mathrm{~h}$ enzymatic hydrolysis were analyzed by HPLC using the ABEE (4-aminobenzoic acid ethyl ester) derivation method described by Yasuno et al. (1997). Hydrolysis experiments were carried out in triplicates.

\subsection{Calculation method of the degree of synergy}

The calculation of the degree of synergy $(D S)$ was determined using the equation (Andersen et al., 2008):

$$
D S=Y_{1+2} /\left(\alpha Y_{1}+\beta Y_{2}\right) \text { or } D S=Y_{1+2+3} /\left(\alpha Y_{1}+\beta Y_{2}+\gamma Y_{3}\right)
$$

where $\alpha, \beta$ and $\gamma$ correspond to the mass ratios of the enzymes. For example, for the binary mixture $\alpha$ and $\beta$ are $0.5 . Y_{1+2}$ represents the glucan or xylan conversion of the mixture by two enzymes working simultaneously, whereas $Y_{1}$ and $Y_{2}$ indicate the glucan or xylan conversion achieved by each enzyme working individually. When XynZ-C, Xyn11A and Cel6A are mixed together in the reaction, the $\alpha$ and $\beta$ values are 0.25 and the $\gamma$ value is 0.5 , respectively. $Y_{1+2+3}$, indicates the glucan or xylan conversion of the mixture by the three enzymes working simultaneously, whereas $Y_{1}, Y_{2}$ and $Y_{3}$ indicate the glucan or xylan conversion achieved by each enzyme working individually. 


\section{Results and Discussion}

\subsection{Effect of pretreatment methods on chemical composition and physical structure of bagasse}

Different pretreatment methods with distinct mechanisms usually have different effects on the same biomass. The chemical compositions of unpretreated, PAA pretreated and [Emim][OAc] pretreated bagasse, are summarized in Table 1. After PAA and $[$ Emim $][\mathrm{OAc}]$ pretreatments the cellulose percentage did not show any clear change. Nevertheless, $5.8 \%$ of lignin and $11.7 \%$ of hemicellulose (xylan and arabinan) were removed from the bagasse after PAA pretreatment. Zhao et al. (2007) reported that $80 \%$ of lignin was removed under conditions of $50 \%$ PAA charge, $1 / \mathrm{s}$ ratio $6: 1,80{ }^{\circ} \mathrm{C}$ for $2 \mathrm{~h}$. However, $59.6 \%$ of the hemicellulose was removed at the same time. In our study, increasing the amount of water diluted the concentration of PAA and aided the preservation of the hemicellulose. For comparison purposes, [Emim][OAc] pretreatment was carried out under the same conditions. The results showed that, in this case, the [Emim][OAc] pretreatment method removed more lignin (26.7\%) than the PAA pretreatment and, interestingly, it retained a large amount of the hemicellulose (33.7\%), especially arabinan (7.2\%). However, this phenomenon was not observed if the [Emim][OAc] was used under high temperature conditions, such as $120^{\circ} \mathrm{C}$ (Qiu et al., 2012).

The results of the yield of regenerated biomass (YRB) indicated that a higher solid content $(79 \%)$ was preserved after $[\mathrm{Emim}][\mathrm{OAc}]$ pretreatment when compared with $63 \%$ preservation following PAA pretreatment (Fig. 1a). Few monosaccharides were dissolved in PAA and [Emim][OAc] regenerated water (data not shown). After acid 
hydrolysis, long soluble oligomers were degraded to monosaccharides, and thus the loss of saccharides with respect to the percentage of total glucan and xylan of untreated bagasse in regenerated water after PAA and [Emim] [OAc] pretreatment were measured (Fig. 1b). After PAA pretreatment, 10.8\% of glucan and $20.9 \%$ of xylan from untreated bagasse were dissolved, whereas [Emim][OAc] pretreatment gave a lower loss of glucan (4.3\%) and xylan (2.8\%). This result can be explained by the different mechanisms by which PAA and IL pretreatments disrupt the structure of cellulose. IL pretreatment dissolves cellulose by forming new hydrogen bonds with cellulose (Brandt et al., 2013; Uju et al., 2013; Yoon et al., 2012). As a result, more glucan remains in the liquid phase after IL pretreatment. However, the mechanism of PAA degradation of lignocellulosic biomass involves the selective attack of the hydroxonium ion on lignin. As a result, partial hemicellulose content, cross-linked with lignin, is removed (Zhao et al., 2007).

The effect of PAA and [Emim][OAc] pretreatments on the structure of bagasse was evaluated by SEM and XRD analyses. After both PAA and [Emim][OAc] pretreatments, the biomass surface and fibrils became rough and disordered, which could be due to the removal of lignin and part of the carbohydrates. Compared with the significantly altered surface of PAA pretreated bagasse, the surface of bagasse after [Emim][OAc] pretreatment preserved the major microfibrous cellulose structure, however, the surface was disrupted (Fig. S2). As shown in Fig. S3, the XRD peak pattern from untreated to [Emim][OAc] pretreated bagasse showed clear changes. The (101) peak of [Emim][OAc] pretreated bagasse disappeared and the (002) peak became broad. This observation is similar to a previous report from Qiu et al. (2012), suggesting the expansion of the cellulose I lattice and the disruption of hydrogen bonds between 
cellulose fibers (Cheng et al., 2011). Based on the XRD pattern, cellulose crystallinity $(\mathrm{CCr})$, which represents the ratio of the crystalline cellulose to the whole cellulose part, was calculated. The $C C r$ value of unpretreated bagasse was 136, whereas [Emim] [OAc] pretreated bagasse gave a $C C r$ value of 68 and PAA pretreated bagasse gave a value of 116. The decrease in the $C C r$ value indicates that part of crystalline cellulose changed form to amorphous cellulose following pretreatment. The lower $C C r$ value of $[$ Emim $][\mathrm{OAc}]$ pretreated bagasse indicates that more amorphous cellulose was recovered after pretreatment and may facilitate enzymatic hydrolysis efficiency (Cheng et al., 2011; Yoon et al., 2012).

\subsection{Production of total sugars from pretreated bagasse}

Enzymatic hydrolysis of PAA and [Emim][OAc] pretreated bagasse by Cel6A, XynZ-C and Xyn11A, solely or mixed together at a fixed loading of $2 \mathrm{mg}$ protein/g bagasse was carried out (Fig. 1c and Fig. S4). In general, the results showed that PAA pretreated bagasse released more reducing sugars by enzymatic hydrolysis when compared with the amount of reducing sugars released from [Emim][OAc] pretreated bagasse. After $72 \mathrm{~h}$ hydrolysis, 1.4-fold more reducing sugars were released from PAA pretreated bagasse than $[$ Emim $][\mathrm{OAc}]$ pretreated bagasse when the three enzymes were used in combination. The reducing sugars are a mixture of several kinds of sugars with reducing ends, including glucose, xylose, cellobiose and so on. Since there are several different potential applications of hexoses and pentoses, and to understand the synergistic effect of cellulase and xylanase on C-5 and C-6 sugars production, further determination of monosaccharides and oligosaccharides released in hydrolysates was performed. 
The soluble sugars released from pretreated bagasse after enzymatic hydrolysis by individual or combined enzymes for $72 \mathrm{~h}$ were analyzed by HPLC. Note that higher polymerized oligomers have not been determined (Fig. 2). The products consisted mostly of cellobiose, xylobiose and xylotriose, with small quantities of glucose and xylose detected. In general, Cel6A converted only a minor amount of glucan from both PAA and [Emim][OAc] pretreated bagasse, even though it has a CBM. The absence of other major components, such as $\beta$-glucosidase and $\mathrm{CBH}$, appears to affect the efficiency of the endoglucanase, even though the endoglucanase has a CBM (Gomez del Pulgar and Saadeddin, 2014; Harris et al., 2014). In the case of PAA pretreated bagasse, glucan conversions by all the enzymatic combinations were low $(<3 \%)$ for the tested conditions. Surprisingly, higher glucan conversion was observed for [Emim][OAc] pretreated bagasse (Fig. 2b). Cel6A alone converted 4\% of glucan from [Emim][OAc] pretreated bagasse, which was four times higher than from PAA pretreated bagasse. This result reflects the impact of cellulose crystallinity in the conversion of glucan during the hydrolysis process. [Emim][OAc] pretreated bagasse with low crystallinity was converted by a higher value than PAA pretreated bagasse, because the endoglucanase randomly cut the cellulose chain from the amorphous region (Harris et al., 2014). Following the addition of xylanase(s) into cellulase, the glucan conversion from PAA pretreated bagasse increased by $>$ 2-fold, even though the amount of Cel6A had been halved. For [Emim] $[\mathrm{OAc}]$ pretreated bagasse, combining Cel6A with an endoxylanase(s) gave a 1.5-fold improvement in conversion. The highest glucan conversion, $8 \%$, was reached when using the ternary mixture at $72 \mathrm{~h}$ from $[\mathrm{Emim}][\mathrm{OAc}]$ pretreated bagasse. Similarly, for PAA pretreated bagasse, the highest glucan conversion, $2.5 \%$, was also observed when using the ternary mixture. Since XynZ-C and Xyn11A only released 
little cellobiose or glucose from pretreated bagasse, the improvement of glucan conversion by mixing Cel6A and xylanase(s) was mostly contributed by the synergistic effect. This observation shows that the removal of xylan favors the action of Cel6A. In the case of xylan conversion, at least 2-fold more xylan was converted from PAA pretreated bagasse than from [Emim][OAc] pretreated bagasse by the same enzyme(s). This observation is possibly a result of the high arabinose percentage in [Emim][OAc] pretreated bagasse inhibiting the xylanases. In non-woody biomass, arabinose is generally present as a substituent in arabinoxylans and is tightly connected to other hemicellulose components (Gírio et al., 2010). Thus, the high percentage of arabinose in the substrate likely protects the substrate from xylanase activity, and this may explain the observed low xylan conversion from [Emim][OAc] pretreated bagasse. XynZ-C without the CBM converted similar amounts of xylan from PAA pretreated bagasse as Xyn11A; however, in the case of [Emim][OAc] pretreated bagasse, Xyn11A achieved a 1.7-fold higher xylan conversion when compared with the results using XynZ-C. This result demonstrates that the presence of a CBM appears to play an important role in xylan conversion. The CBM facilitates the interaction of the enzyme with the substrate and therefore increases the local concentration of the enzyme on the insoluble biomass substrate. In the present study, Xyn11A with a XBM could potentially increase the accessibility of the catalytic domain of Xyn11A to the substrate. On the other hand, the low arabinan amount in PAA pretreated bagasse indicates the high accessibility of the substrate to xylanase. Thus, even XynZ-C without any binding module could convert similar amounts of xylan from PAA pretreated bagasse compared to Xyn11A. Improving the conversion of xylan after xylanase replacement was not as apparent as glucan conversion. For PAA pretreated bagasse, after combining with Cel6A, Xyn11A 
converted less xylan (21.3\%) than the enzyme achieved individually (25.9\%).

Correspondingly, in the case of [Emim] [OAc] pretreated bagasse, the combination of Cel6A and Xyn11A yielded a similar xylan conversion amount when compared with the amount converted using Xyn11A alone. This is probably because of the competition of the two binding modules. Finally, the ternary mixture converted the maximum amount of xylan (37.8\% and $16.1 \%)$ from PAA and [Emim][OAc] pretreated bagasse, respectively.

\subsection{Synergy between endoxylanases and endoglucanase on pretreated bagasse}

The addition of endoxylanases into endoglucanases improved both glucan and xylan conversions. The relationships between xylan removal and glucan conversion for the different enzyme combinations (Cel6A, XynZ-C and/orXyn11A) are shown in Fig.

3. This linear relationship confirmed the postulate that the removal of xylan from the cellulose matrix had a positive effect on glucan conversion, because the hemicellulose is highly cross-linked by diferulic bridges and cellulose fibrils are likely incorporated (Kumar and Wyman, 2009b; Saha, 2003; Selig et al., 2008; Zhang and Viikari, 2014). The slope of [Emim][OAc] pretreated bagasse was steeper than PAA pretreated bagasse. [Emim][OAc] pretreated bagasse presented a lower $C C r$ value, which indicates that the more amorphous cellulose forms are exposed the easier the cellulase hydrolysis, and thus high cellulose conversion could be observed. We hypothesize that for the [Emim][OAc] pretreated bagasse, once a small amount of xylan is removed the conversion may yield high cellulose conversion. In contrast, PAA pretreated bagasse exposed cellulose that was more crystalline, and thus only a small incremental improvement in cellulose conversion, induced by xylan removal, was observed. 
The observed improvement of glucan and xylan conversion in the hydrolysis of pretreated bagasse, after combining Cel6A and xylanase(s) demonstrates the synergism between cellulase and xylanase(s). The calculation of the degree of synergy is a direct way to evaluate the synergism between enzymes. In general, when the value of $D S$ is higher than 1, the synergism between cellulase and xylanase is active. In the present study, $D S$ was calculated based on the percentage of glucan and xylan conversion from different pretreated bagasses after $72 \mathrm{~h}$ of hydrolysis (Fig. 4). The $D S$ values for glucan conversion were higher than the $D S$ values for xylan conversion in the hydrolysis of both PAA and [Emim][OAc] pretreated bagasse. This indicates that the synergistic effect between endoglucanase and endoxylanase is stronger for glucan conversion than for the conversion of xylan.

Usually, the physical structure and chemical composition of the substrate is noticeably altered after pretreatment (Alvira et al., 2010; Brandt et al., 2013; Harris et al., 2014). Previous reports showed that pretreatment methods significantly affect the synergism of cellulase and xylanase (Kumar and Wyman, 2009b; Li et al., 2014). In the present study, all the combinations showed a more evident synergism (over 3.4) for glucan conversion in the hydrolysis of PAA pretreated bagasse, whereas a higher $D S$ value for xylan conversion was achieved from hydrolyzing the [Emim][OAc] pretreated bagasse. For PAA pretreated bagasse, a relatively larger amount of xylan was converted after the addition of xylanase(s) (Fig. 2a); thus, because more degradable cellulose fractions were exposed, the $D S$ values for glucan conversion were high. However, because of the high crystallinity of cellulose in PAA pretreated bagasse, low glucan conversion was observed. Only a small amount of xylan was exposed from the interaction between endoglucanase and endoxylanase, and as a consequence the $D S$ 
values for xylan conversion were low. In the case of [Emim][OAc] pretreated bagasse, because of the highly cross-linked structure of hemicellulose but low crystallinity of cellulose, a higher $D S$ value for xylan conversion and a lower $D S$ value for glucan conversion were observed.

Furthermore, it was reported that the molecular structure of the enzyme could also affect the synergism. The lack of a CBM in xylanase Xyn11A from $C$. flavigena was shown to decrease the synergy between a xylanase and a cellulase (endoglucanase Cel7B from T. reesei) in the hydrolysis of alkaline pretreated bagasse (Pavón-Orozco et al., 2012). In this study, for PAA pretreated bagasse, the combination of Cel6A + XynZ-C achieved a lower $D S$ for glucan conversion (3.4), but a higher $D S$ for xylan conversion (1.9) when compared with the results of combining Cel6A + Xyn11A, which had $D S$ values for glucan and xylan conversion of 4.7 and 1.6, respectively. Likewise, for [Emim] $[\mathrm{OAc}]$ pretreated bagasse, the combination of Cel6A and XynZ-C reached similar $D S$ values for glucan and xylan conversion of 2.7 and 2.6, respectively. The combination Cel6A + Xyn11A reached a higher $D S$ value for glucan conversion (3.4) than the combination of Cel6A + XynZ-C, but a lower $D S$ value for xylan conversion (1.9). As previously discussed, Xyn11A with a XBM converted more xylan than XynZ-C, which does not have a substrate binding module. Thus, more cellulose was exposed from the combination of Cel6A + Xyn11A. This combination showed high DS values for glucan conversion for both PAA and [Emim][OAc] pretreated bagasse. Correspondingly, glucan dissolution could create cleavage sites for xylanase. Thus, for XynZ-C without a CBM, this dissolution may potentially increase its efficiency and may explain the higher $D S$ value for xylan conversion by the Cel6A + XynZ-C combination. On the other hand, for Xyn11A with a XBM, this improvement would not 
be that apparent, hence, the $D S$ value for xylan conversion was lower. In conclusion, the presence of a substrate-binding module within a xylanase provides greater synergism for glucan conversion; the cellulose dissolution favored more the action of xylanase without a binding module. Not surprisingly, the highest $D S$ values, for both glucan and xylan conversion, were achieved by the ternary mixture in the hydrolysis of PAA or $[$ Emim] $]$ OAc] pretreated bagasse.

In summary, in the hydrolysis of PAA or [Emim][OAc] pretreated bagasse, it appears that not only the hemicellulose components and structure, and the cellulose crystallinity but also the molecular structure of the enzyme should be carefully considered to attain optimal synergism between a xylanase and a cellulase. These observations provide the basis for understanding the synergism of cellulases and xylanases on a pretreated lignocellulosic biomass and the development of more elaborated enzyme mixtures for the creation of a cost-effective and highly efficient biorefinery industry.

\section{Conclusions}

Synergism between cellulase and xylanase in the hydrolysis of bagasse was affected by structural and compositional differences between the substrates resulting from the different pretreatments. PAA pretreatment removed part of hemicellulose but left more crystalline cellulose, resulting in a high degree of synergy for glucan conversion. In contrast, [Emim][OAc] pretreatment likely disrupted less hemicellulose-cellulose associations but generated more amorphous cellulose, resulting in a high degree of synergy for xylan conversion. The molecular structure of enzymes also affected the synergism. Owing to the crosslinking of hemicellulose and cellulose, 
the removal of xylan favored glucan conversion, and vice versa.

\section{Acknowledgements}

This research was supported by the Advanced Low Carbon Technology Research and Development Program (ALCA) from the Japan Science and Technology Agency (JST). L. Jia thanks the China Scholarship Council (grant number: 201306300044). 


\section{References}

1. Alvira, P., Tomás-Pejó, E., Ballesteros, M., Negro, M.J., 2010. Pretreatment technologies for an efficient bioethanol production process based on enzymatic hydrolysis: A review. Bioresour. Technol. 101, 4851-4861.

2. Andersen, N., Johansen, K.S., Michelsen, M., Stenby, E.H., Krogh, K.B.R.M., Olsson, L., 2008. Hydrolysis of cellulose using mono-component enzymes shows synergy during hydrolysis of phosphoric acid swollen cellulose (PASC), but competition on Avicel. Enzyme Microb. Technol. 42, 362-370.

3. Brandt, A., Gräsvik, J., Hallett, J.P., Welton, T., 2013. Deconstruction of lignocellulosic biomass with ionic liquids. Green Chem. 15, 550.

4. Chandel, A.K., da Silva, S.S., Carvalho, W., Singh, O. V., 2012. Sugarcane bagasse and leaves: foreseeable biomass of biofuel and bio-products. J. Chem. Technol. Biotechnol. 87, 11-20.

5. Cheng, G., Varanasi, P., Li, C., Liu, H., Melnichenko, Y.B., Simmons, B.A., Kent, M.S., Singh, S., 2011. Transition of cellulose crystalline structure and surface morphology of biomass as a function of ionic liquid pretreatment and its relation to enzymatic hydrolysis. Biomacromolecules 12, 933-941.

6. Davis, R., Tao, L., Tan, E.C.D., Biddy, M.J., Beckham, G.T., Scarlata, C., 2013. Process design and economics for the conversion of lignocellulosic biomass to hydrocarbons $\square$ : dilute-acid and enzymatic deconstruction of biomass to sugars and biological conversion of sugars to hydrocarbons. NREL, Golden, CO.

7. Gírio, F.M., Fonseca, C., Carvalheiro, F., Duarte, L.C., Marques, S., Bogel-Łukasik, R., 2010. Hemicelluloses for fuel ethanol: A review. Bioresour. Technol. 101, 4775-4800.

8. Gomez del Pulgar, E.M., Saadeddin, A., 2014. The cellulolytic system of Thermobifida fusca. Crit. Rev. Microbiol. 40, 236-247.

9. Gonçalves, G.A.L., Takasugi, Y., Jia, L., Mori, Y., Noda, S., Tanaka, T., Ichinose, H., 
Kamiya, N., 2015. Synergistic effect and application of xylanases as accoessory enzymes to enhance the hydrolysis of pretreated bagasse. Enzyme Microb. Technol. http://dx.doi.org/10.1016/j.enzmictec.2015.01.007

10. Harris, P. V, Xu, F., Kreel, N.E., Kang, C., Fukuyama, S., 2014. New enzyme insights drive advances in commercial ethanol production. Curr. Opin. Chem. Biol. 19, 162-170.

11. Hu, J., Arantes, V., Pribowo, A., Saddler, J.N., 2013. The synergistic action of accessory enzymes enhances the hydrolytic potential of a "cellulase mixture" but is highly substrate specific. Biotechnol. Biofuels 6, 112.

12. Kumar, R., Hu, F., Hubbell, C. a, Ragauskas, A.J., Wyman, C.E., 2013. Comparison of laboratory delignification methods, their selectivity, and impacts on physiochemical characteristics of cellulosic biomass. Bioresour. Technol. 130, $372-381$.

13. Kumar, R., Wyman, C., 2009a. Effects of cellulase and xylanase enzymes on the deconstruction of solids from pretreatment of poplar by leading technologies. Biotechnol. Prog. 25, 302-314.

14. Kumar, R., Wyman, C.E., 2009b. Effect of xylanase supplementation of cellulase on digestion of corn stover solids prepared by leading pretreatment technologies.

Bioresour. Technol. 100, 4203-4213.

15. Li, J., Zhou, P., Liu, H., Xiong, C., Lin, J., Xiao, W., Gong, Y., Liu, Z., 2014. Synergism of cellulase, xylanase, and pectinase on hydrolyzing sugarcane bagasse resulting from different pretreatment technologies. Bioresour. Technol. 155, 258265.

16. Lv, S., Yu, Q., Zhuang, X., Yuan, Z., Wang, W., Wang, Q., Qi, W., Tan, X., 2013. The influence of hemicellulose and lignin removal on the enzymatic digestibility from sugarcane bagasse. BioEnergy Res. 6, 1128-1134.

17. Miller, G.L., 1959. Use of dinitrosalicylic acid reagent for determination of reducing sugar. Anal. Chem. 31, 426-428. 
18. Mora-Pale, M., Meli, L., Doherty, T. V, Linhardt, R.J., Dordick, J.S., 2011. Room temperature ionic liquids as emerging solvents for the pretreatment of lignocellulosic biomass. Biotechnol. Bioeng. 108, 1229-1245.

19. Pavón-Orozco, P., Santiago-Hernández, A., Rosengren, A., Hidalgo-Lara, M.E., Stålbrand, H., 2012. The family II carbohydrate-binding module of xylanase CflXyn11A from Cellulomonas flavigena increases the synergy with cellulase TrCel7B from Trichoderma reesei during the hydrolysis of sugar cane bagasse. Bioresour. Technol. 104, 622-630.

20. Qiu, Z., Aita, G.M., Walker, M.S., 2012. Effect of ionic liquid pretreatment on the chemical composition, structure and enzymatic hydrolysis of energy cane bagasse. Bioresour. Technol. 117, 251-256.

21. Saha, B.C., 2003. Hemicellulose bioconversion. J. Ind. Microbiol. Biotechnol. 30, 279-291.

22. Sant'Ana da Silva, A., Lee, S.-H., Endo, T., Bon, E.P.S., 2011. Major improvement in the rate and yield of enzymatic saccharification of sugarcane bagasse via pretreatment with the ionic liquid 1-ethyl-3-methylimidazolium acetate ([Emim] [Ac]). Bioresour. Technol. 102, 10505-10509.

23. Selig, M.J., Knoshaug, E.P., Adney, W.S., Himmel, M.E., Decker, S.R., 2008. Synergistic enhancement of cellobiohydrolase performance on pretreated corn stover by addition of xylanase and esterase activities. Bioresour. Technol. 99, 4997-5005.

24. Sluiter, A., Hames, B., Ruiz, R., Scarlata, C., Sluiter, J., Templeton, D., 2008a. Determination of Ash in Biomass. NREL, Golden, CO.

25. Sluiter, A., Hames, B., Ruiz, R., Scarlata, C., Sluiter, J., Templeton, D., Crocker, D., 2011. Determination of Structural Carbohydrates and Lignin in Biomass. NREL, Golden, CO.

26. Sluiter, A., Ruiz, R., Scarlata, C., Sluiter, J., Templeton, D., 2008b. Determination of extractives in biomass. NREL, Golden, CO. 
27. Uju, Abe, K., Uemura, N., Oshima, T., Goto, M., Kamiya, N., 2013. Peracetic acid-ionic liquid pretreatment to enhance enzymatic saccharification of lignocellulosic biomass. Bioresour. Technol. 138, 87-94.

28. Xu, F., Shi, Y.-C., Wang, D., 2013. X-ray scattering studies of lignocellulosic biomass: a review. Carbohydr. Polym. 94, 904-917.

29. Yasuno, S., Murata, T., Kokubo, K., Yamaguchi, T., Kamei, M., 1997. Two-mode analysis by high-performance liquid chromatography of p-aminobenzoic ethyl ester-derivatized monosaccharides. Biosci. Biotech. Biochem 61, 1944-1946.

30. Yoon, L.W., Ang, T.N., Ngoh, G.C., Chua, A.S.M., 2012. Regression analysis on ionic liquid pretreatment of sugarcane bagasse and assessment of structural changes. Biomass and Bioenergy 36, 160-169.

31. Zhang, J., Viikari, L., 2014. Impact of Xylan on Synergistic Effects of Xylanases and Cellulases in Enzymatic Hydrolysis of Lignocelluloses. Appl. Biochem. Biotechnol. 174, 1393-1402.

32. Zhao, X., Wang, L., Liu, D., 2007. Effect of several factors on peracetic acid pretreatment of sugarcane bagasse for enzymatic hydrolysis. J. Chem. Technol. Biotechnol. 82, 1115-1121. 


\section{Figure Captions}

Fig. 1. Comparison of the effect of PAA and [Emim] [OAc] pretreatments on: (a) the yield of regenerated biomass. (b) The percentages of glucan and xylan lost in regenerated water to total glucan and xylan contents in untreated bagasse. (c) Reducing sugars concentration after 3, 6, 12, 24, 48 and $72 \mathrm{~h}$ hydrolysis of PAA pretreated bagasse and [Emim][OAc] pretreated bagasse by a mixture of endoglucanase, Cel6A from T. fusca and endoxylanases, XynZ-C and Xyn11A, from C. thermocellum and T. fusca. The error bars represent standard deviations for three independent trials.

Fig. 2. Glucan conversion and xylan conversion after $72 \mathrm{~h}$ hydrolysis of (a) PAA pretreated bagasse (b) [Emim] [OAc] pretreated bagasse by individual or different combinations of endoglucanase, Cel6A from T. fusca and xylanases, XynZ and Xyn11A, from $C$. thermocellum and T. fusca. For each trial, the enzyme loading was held constant at $2 \mathrm{mg}$ protein/g biomass. The error bars represent standard deviations for three independent trials.

Fig. 3. Relationship between glucan and xylan conversion during the hydrolysis of PAA pretreated bagasse (white symbol) and [Emim][OAc] pretreated bagasse (black symbol) by Cel6A alone (square), Cel6A + XynZ-C (diamond), Cel6A + Xyn11A (triangle) and Cel6A + XynZ-C + Xyn11A (circle) after 72 h hydrolysis

Fig. 4. Degree of synergy of endoglucanase and xylanase(s) for the conversion of glucan (white bar) and xylan (black bar) on (a) PAA pretreated bagasse and (b) [Emim] [OAc] pretreated bagasse after $72 \mathrm{~h}$ of hydrolysis. 


\section{Tables}

\section{Table 1}

Chemical composition of unpretreated, PAA and [Emim] [OAc] pretreated bagasse and the yield of regenerated bagasse.

\begin{tabular}{lccc}
\hline & Unpretreated $^{\mathrm{a}}$ & PAA pretreated $^{\mathrm{a}}$ & [Emim][OAc] pretreated \\
\hline Glucan & $37.7 \pm 1.1$ & $38.6 \pm 0.4$ & $38.9 \pm 1.6$ \\
Xylan & $22.0 \pm 5.6$ & $21.0 \pm 0.7$ & $26.5 \pm 1.0$ \\
Arabinan & $2.8 \pm 0.8$ & $0.9 \pm 0.3$ & $7.2 \pm 0.6$ \\
Ash & $3.7 \pm 0.4$ & $2.7 \pm 0.5$ & $2.3 \pm 0.6$ \\
Extractives & $13.1 \pm 1.6$ & $11.5 \pm 0.4$ & $9.3 \pm 0.9$ \\
Acid insoluble lignin & $20.2 \pm 0.8$ & $14.9 \pm 2.5$ & $12.0 \pm 3.0$ \\
Acid soluble lignin & $2.3 \pm 0.1$ & $6.3 \pm 0.3$ & $4.5 \pm 0.1$ \\
\hline
\end{tabular}

${ }^{\mathrm{a}}$ Gonçalves et al., 2015 
(a)

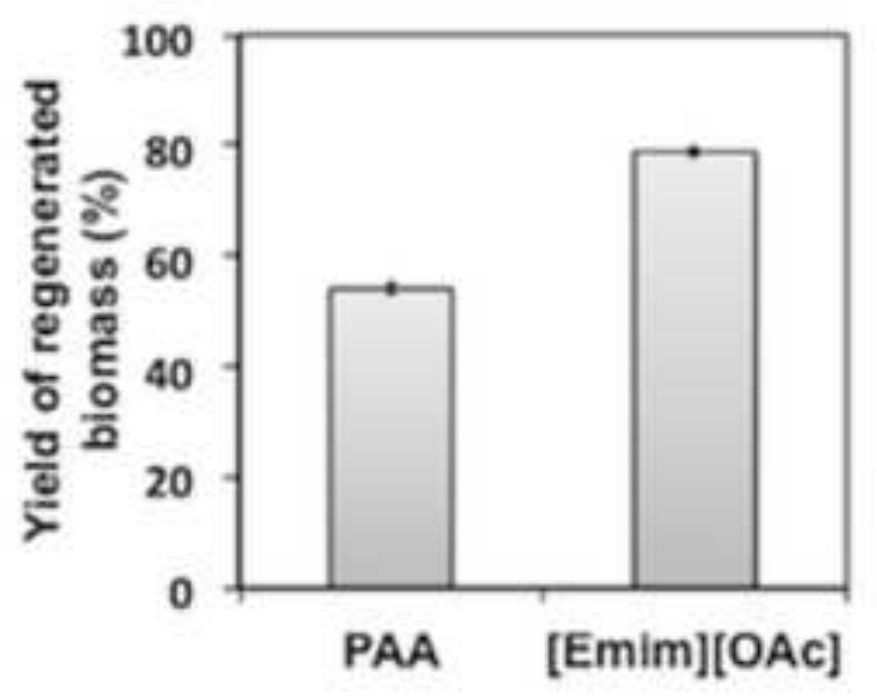

(b)

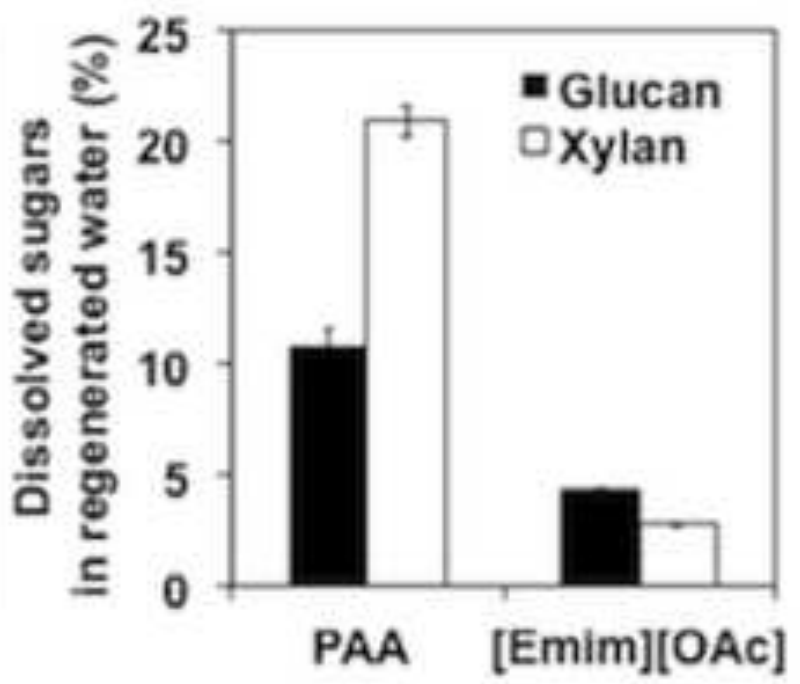

(c)

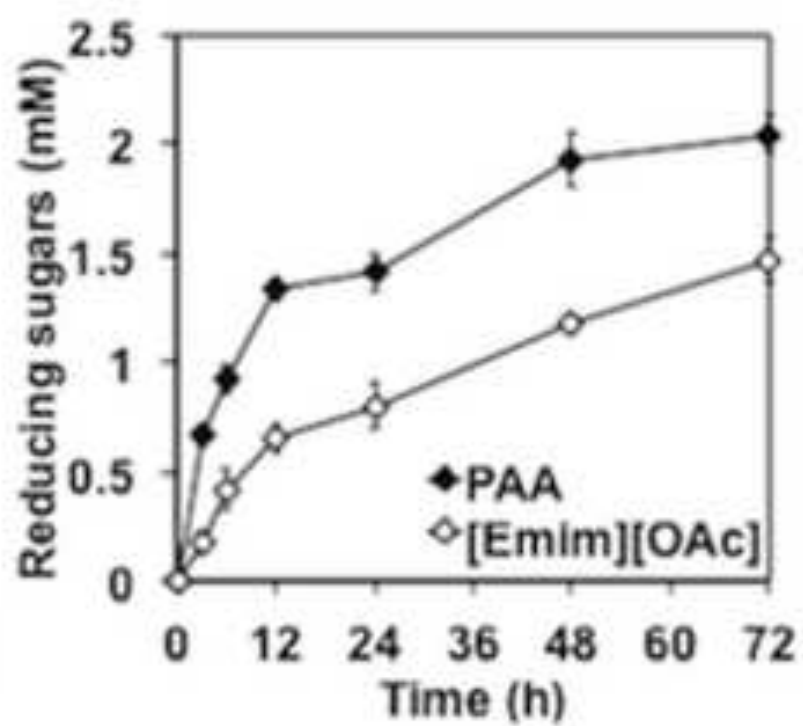


(a)
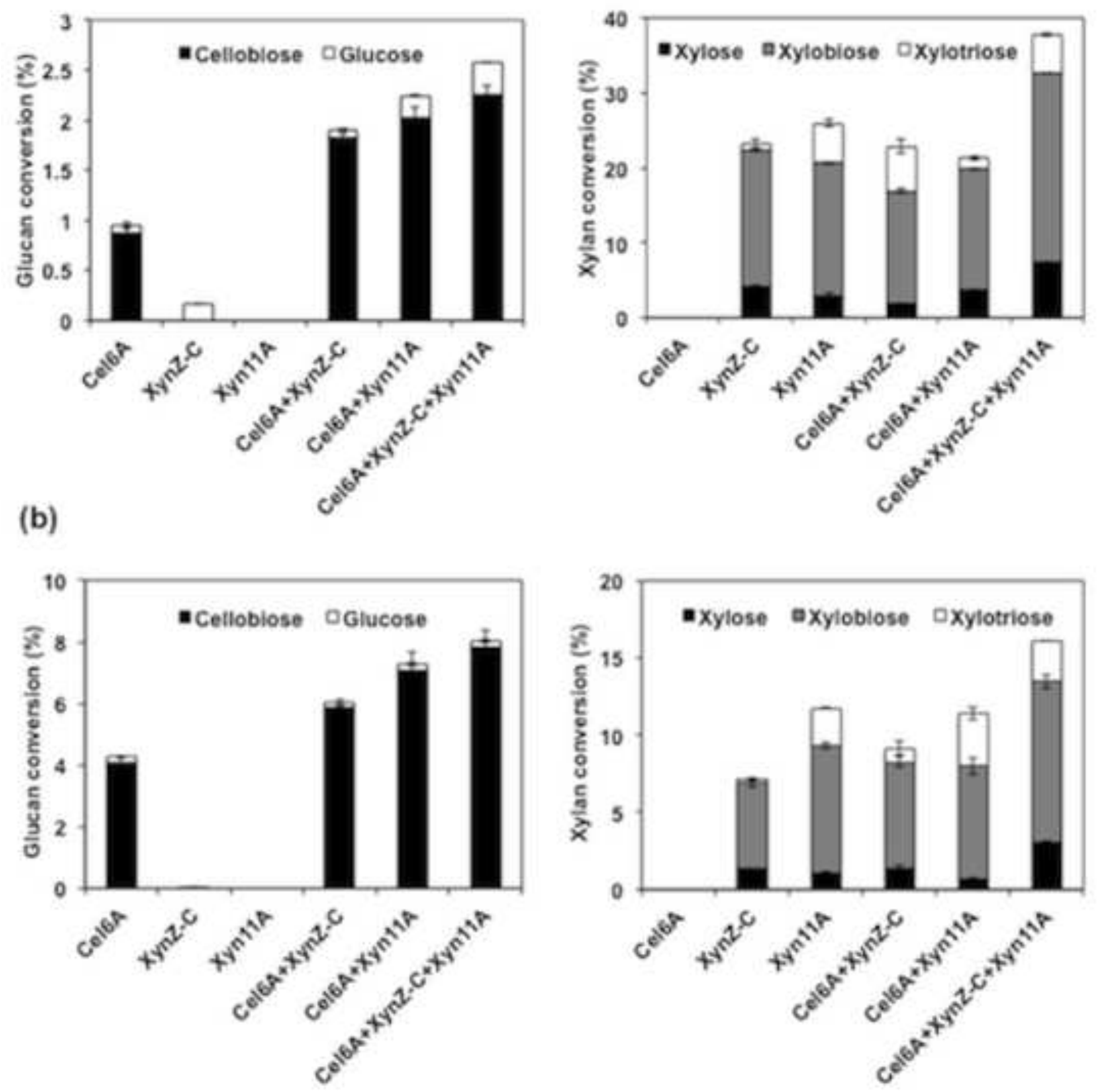


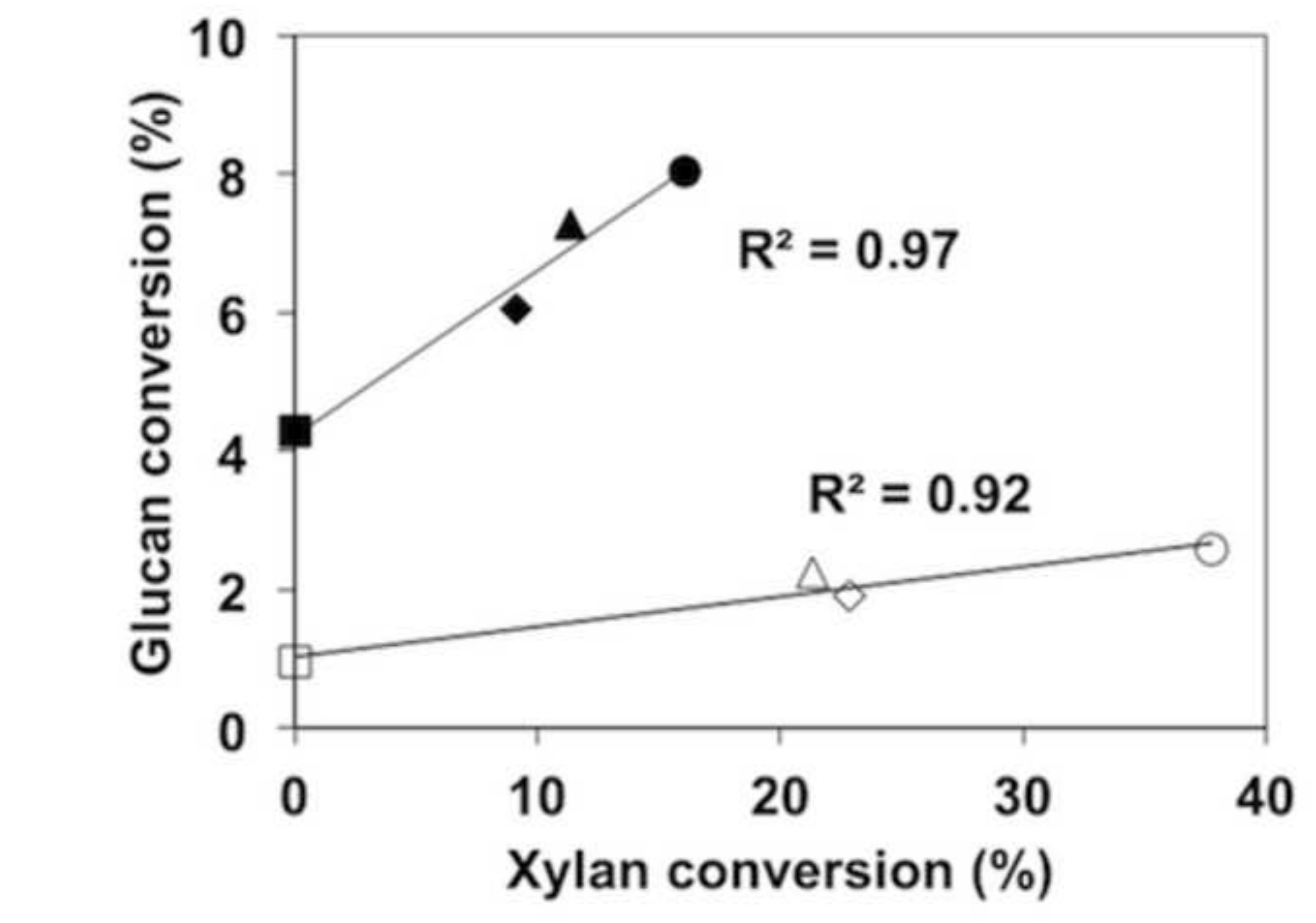


(a)

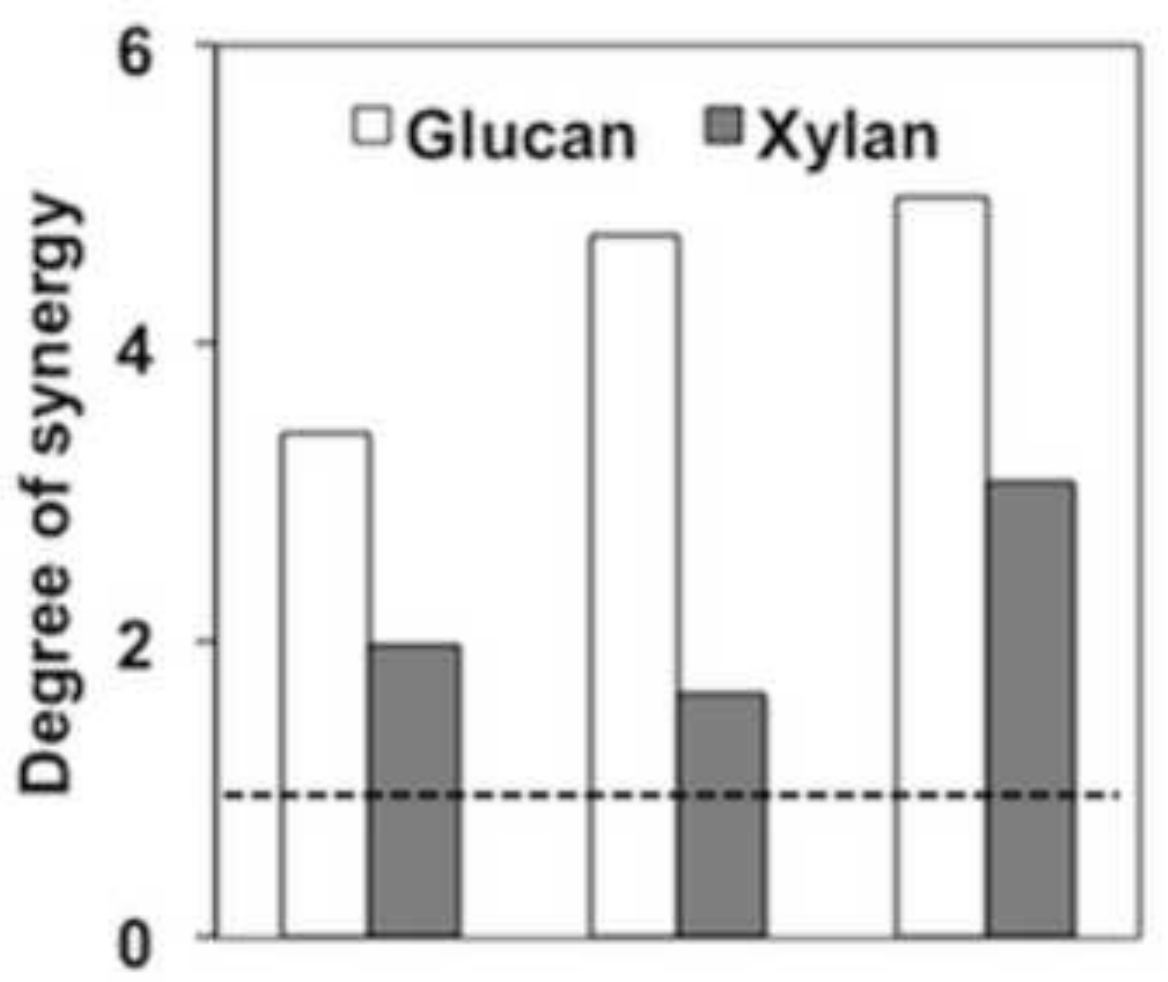

Cel6A

Xynz-C +

Xyn11A - (b)

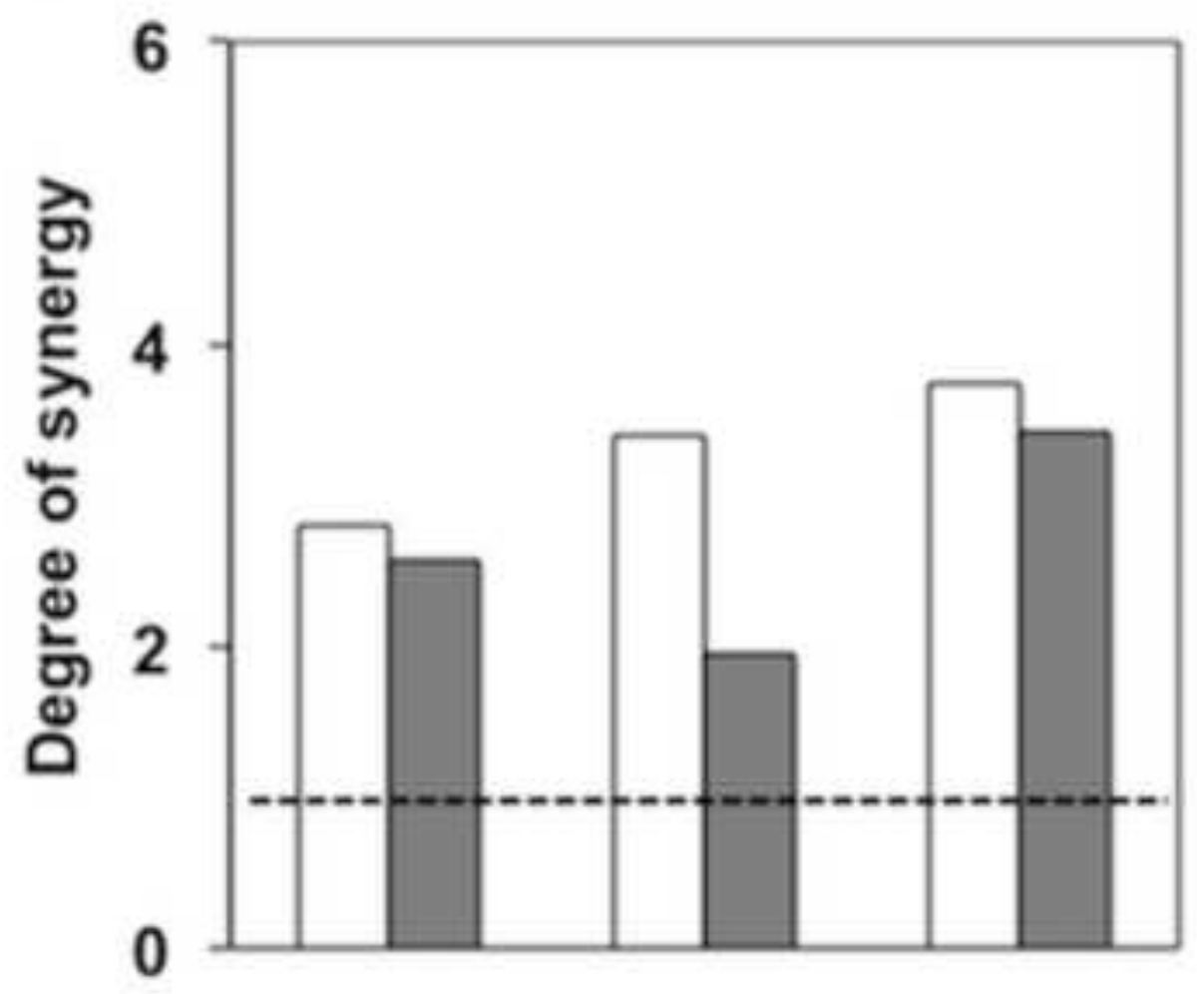

$+$

-

$+$
$+$

$+$

$+$
Cel6A

XynZ-C

$+\quad$ Xyn11A -

$+$

$+$ 


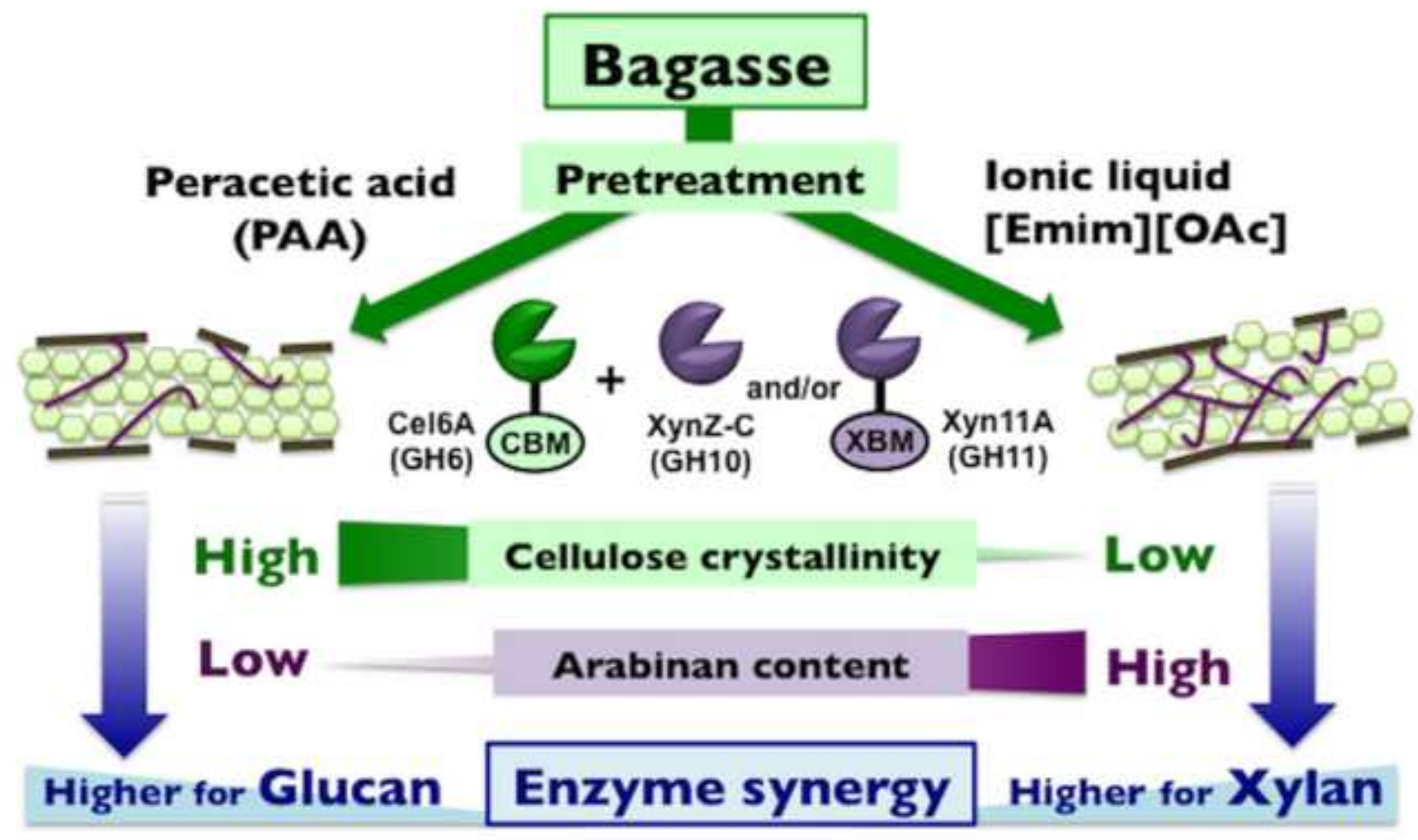

